\title{
Tratamiento integral de fisura palatina con injerto de lengua. Reporte de caso
}

\section{Sinus floor elevation with a lateral window technique and simultaneous dental implants placement: case report.}

\author{
Leiva Noemí ${ }^{1}$, Bravo Pablo ${ }^{1 *}$, Nieto Eugenio ${ }^{2}$, Cazenave Lissette ${ }^{1}$.
}

1. Universidad de Chile, Chile

2. Universidad Mayor, Chile

${ }^{*}$ Correspondencia autor: Dr. Pablo Bravo Alegría. I Fono: +569 93199291 | E-mail: pablo.bravo.a@gmail.com Trabajo recibido el 24/04/2017. Aprobado para su publicación el 27/08/2017

\section{RESUMEN}

La fisura labio palatina (FLP) es una alteración del desarrollo, congénita, de etiología desconocida. La fístula oronasal es la complicación más común de la reparación del paladar con fisura. Los problemas más comunes incluyen hipernasalidad al hablar y el pase de fluidos y comida hacia la cavidad nasal. El caso corresponde a un paciente de 16 años de edad, sexo masculino, con fístula buconasal de 2,1 cm de diámetro mayor en sentido transversal, como secuela de FLP unilateral derecha. Además relata problemas en la alimentación, traspaso de fluidos a la cavidad nasal, problemas de habla por insuficiencia velo faríngea (IVF) valor 9, alteraciones en sus relaciones interpersonales y baja autoestima. Se realizó el injerto de lengua en fístula buconasal, tratamiento de ortodoncia y rehabilitación oral para dar solución estética y funcional. El éxito del tratamiento integral le permitió al paciente mejorar sus relaciones sociales debido a una mayor autoestima.

\section{PALABRAS CLAVE}

Fisura labio palatina; fístula buconasal; Ortodoncia; Rehabilitación oral; Cirugía plástica; Injerto de lengua.

Rev. Clin. Periodoncia Implantol. Rehabil. Oral Vol. 10(3); 163-165, 2017.

\section{ABSTRACT}

The cleft lip and palate (CLP) is a developmental and congenital anomaly of unknown etiology. The oronasal fistula is the most common complication of the cleft palate reparation. The most common problems are hypernasality on speech and the passage of fluids and food to the nasal cavity. This case is about a sixteen- year-old boy with a $2,1 \mathrm{~cm}$ diameter oronasal fistula due to a right unilateral CLP sequel. He also presented alimentation problems, fluid passage to the nasal cavity, speech defects by velopharyngeal insufficiency (VFI), relationships problems and low self-esteem. A tongue graft in the oronasal fistula, orthodontic treatment and oral rehabilitation were made to give an aesthetic and functional solution. The success of the treatment allowed the patient to improve his relationships due to a greater self-esteem. KEYWORDS

Cleft lip palate; oronasalfistula; Orthodontics; Oral Rehabilitation; Plastic surgery; Tongue graft.

Rev. Clin. Periodoncia Implantol. Rehabil. Oral Vol. 10(3); 163-165, 2017.

\section{INTRODUCCIÓN}

La fisura labio palatina (FLP) es una alteración del desarrollo, congénita, de etiología desconocida, probablemente ligada a factores genéticos y externos. Se forma entre la 4-9 semana de gestación y afecta a 1 de cada 700-750 niños nacidos vivos. Un tercio de las fisuras incluyen las fisuras palatinas, mientras que el resto abarcan aquellas fisuras de labio (unilateral o bilateral) con o sin fisura palatina. Las FLP pueden generar alteraciones dentarias de número, tamaño, forma y posición. La pieza dentaria más afectada es aquella que está próximo a la fisura.(1) Se ha observado que aquellos pacientes que presentan mayor hipoplasia maxilar son aquellos que presentan agenesia del incisivo lateral aunque no excluye a ningún paciente. ${ }^{(2)}$

El objetivo del cierre quirúrgico de la severa fisura palatina será la recuperación funcional permitiendo mejor alimentación y habla. Para el logro de estas metas, será imprescindible el cierre de las tres capas de tejidos involucradas: mucosa nasal, muscular y mucosa oral.(1)
La fístula palatina anterior o fístula oronasal es la complicación más común de la reparación del paladar con fisura, con un rango de incidencia de entre $4 \%$ y $35 \%{ }^{(3,4,5)} \mathrm{A}$ pesar de la mejoría en las técnicas en la reparación del paladar con fisura, la ocurrencia de fístula sigue siendo una posibilidad en algunos casos por una baja calidad de los tejidos del paciente. ${ }^{(6)}$

Los síntomas más comunes incluyen hipernasalidad al hablar debido al escape nasal de aire y el pase de fluidos y comida hacia la cavidad nasal, resultando en una higiene oral deficiente, mal olor y enorme molestia para el paciente.

Cohen y Posnick propusieron las siguientes clasificaciones con base en el tamaño del defecto:

- Hendidura simple. Defecto mínimo en la línea media. Causado comúnmente por una pequeña dehiscencia sobre el paladar duro.

- Fístulas pequeñas. Menos de $1.5 \mathrm{~cm}$ de diámetro; se localiza comúnmente en la línea media. Causado por una pequeña dehiscencia sobre la unión del paladar duro y blando o por una pequeña necrosis en 
los bordes de los colgajos

- Fístulas grandes. Mayor de $1.5 \mathrm{~cm}$ de diámetro y comúnmente causada por necrosis del tercio anterior de los colgajos probablemente por lesión a la arteria palatina, se puede comunicar con la hendidura alveolar. ${ }^{(7)}$

De acuerdo al caso presentado, éste se clasifica como una fístula grande, presentando en su diámetro mayor $2.1 \mathrm{~cm}$

Según Cohen y cols. ellos clasificaban de la siguiente manera:

- Fístulas pequeñas. 1 a $2 \mathrm{~mm}$.

- Fístulas medianas. 3 a $5 \mathrm{~mm}$.

- Fístulas grandes. Mayores a $5 \mathrm{~mm} .^{(3)}$

Las variadas opciones de tratamiento disponibles incluyen colgajos de tejido local, palatoplastía de revisión, colgajos regionales como colgajos de mucosa bucal, colgajos faríngeos, colgajos de lengua, transferencia de tejido libre microvascular (colgajo radial) y finalmente rehabilitación oral y protésica. ${ }^{(8,9,10,11)}$

Aunque comúnmente el cierre de la fístula se establece por el uso de colgajos locales, a veces el sitio y tamaño de la fístula hacen que el uso de estos para su reparación sea una posibilidad remota. La utilización de colgajos de lengua debido a su posición central en el piso de boca movilidad y la diversidad de posicionamiento de los colgajos la hacen un método de elección para el cierre de fístulas palatinas anteriores mayores, más que otros tejidos. ${ }^{(6)}$

Existen dos técnicas para obtener colgajos dorsales de lengua: de base anterior o de base posterior. Los colgajos de base anterior están indicados para defectos del paladar duro, mucosa bucal anterior, piso anterior de la boca y labios; los colgajos de base posterior para defectos de paladar blando, área retromolar y mucosa bucal posterior. ${ }^{(12,13)}$

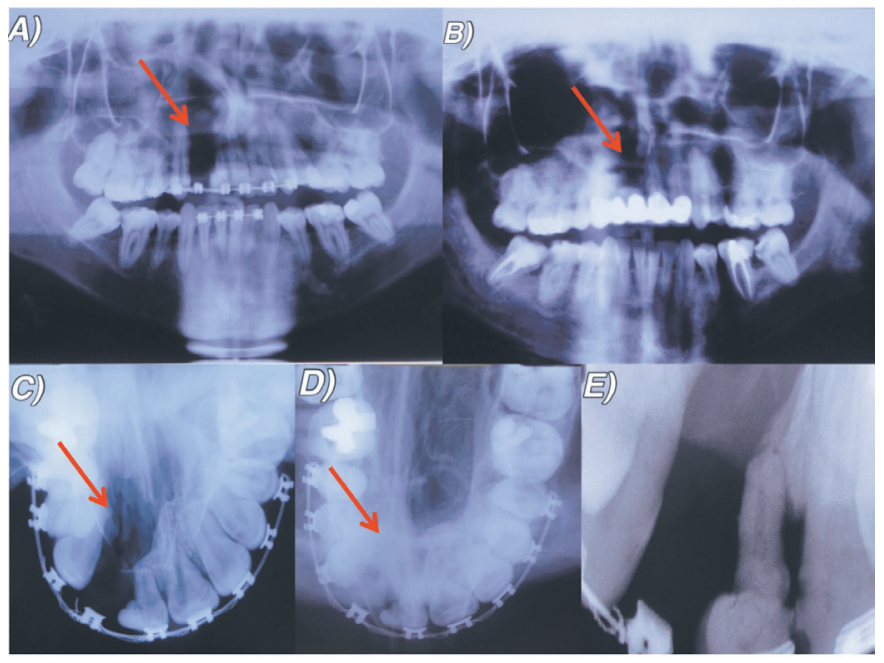

Figura 1. A. Radiografía panorámica de fisura unilateral derecha. Paciente en inicio de tratamiento de ortodoncia. B. Radiografía panorámica luego de tratamiento rehabilitador finalizado. C. Radiografía oclusal de fisura unilateral derecha. D. Radiografía oclusal luego de injerto óseo en zona de fisura palatina. E. Radiografía periapical de pieza 1.1 en relación a zona de la fisura.

\section{CASO CLÍNICO}

Paciente D.C.R de 16 años de edad, sexo masculino, acude a la Unidad de Malformaciones Craneofaciales de la Facultad de Odontología de la Universidad de Chile para tratamiento de Ortodoncia, con fístula buconasa de $2,1 \mathrm{~cm}$ de diámetro en sentido transversal en su diámetro mayor, como secuela de fisura unilateral derecha de labio, paladar y velo. El paciente presenta una clase II esqueletal con falta de desarrollo del tercio medio facial. En sentido sagital presenta una distoclusión molar bilateral y un resalte negativo de $-0.5 \mathrm{~mm}$, en sentido transversal una mordida cruzada bilateral y en sentido vertical un escalón disminuido, con un valor de $0 \mathrm{~mm}$.

El paciente relata que la fístula buconasal le dificulta la alimentación debido al paso de alimentos a través de ella desde la cavidad oral a la cavidad nasal, ocurriendo frecuentemente salida de fluidos a través de las fosas nasales, impidiendo esto la correcta ingesta de bebidas y alimentos de consistencia líquida. Además presenta una insuficiencia velo-faríngea (IVF) 9, lo cual dificulta de manera severa el habla, trayendo consecuentemente alteraciones en su autoestima y relaciones interpersonales.

El paciente fue operado en 4 oportunidades para el cierre del paladar: al año y seis meses, a los 4 años, 9 años y 12 años.

Se realiza interconsulta a Cirugía Plástica para la evaluación del cierre de la fístula y coordinar el inicio del tratamiento de ortodoncia. Este último

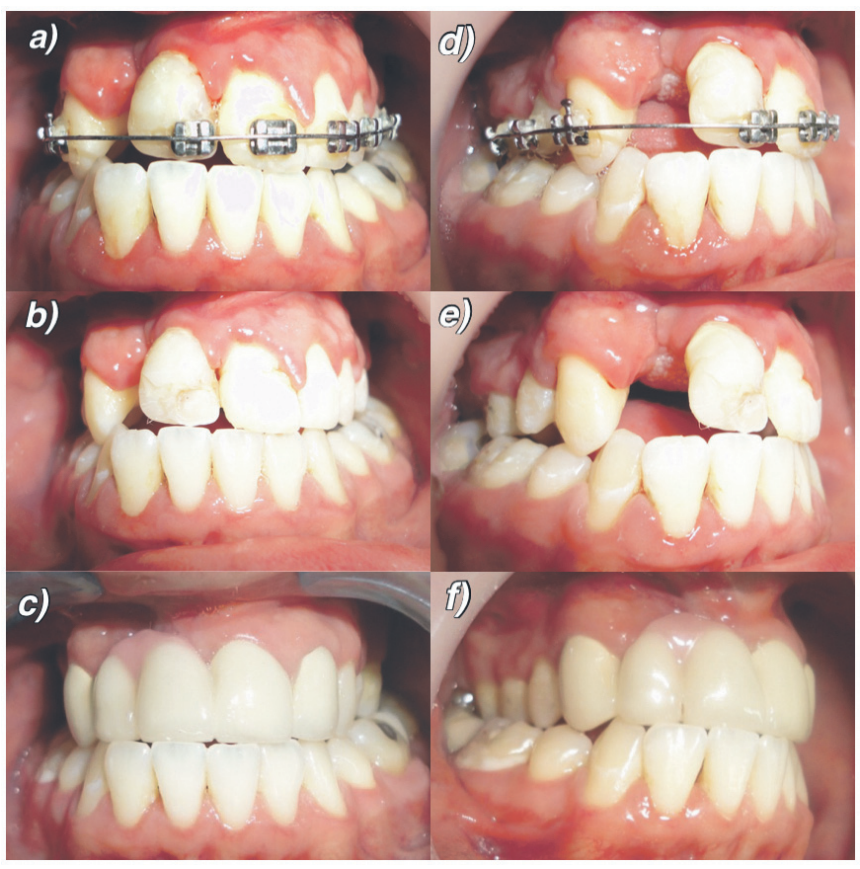

Figura 2. Fotografías frontales de arcadas en oclusión (a-c). Fotografías laterales derechas de arcadas en oclusión (d-f)a. Paciente durante tratamiento de ortodoncia con aparatología fija. b. Paciente al terminar tratamiento de ortodoncia. c. Paciente al finalizar tratamiento rehabilitador mediante prótesis fija plural con pilares en piezas 1.3,2.1 y 2.2, para rehabilitar piezas 1.1 y 1.2 . Nótese además porcelana rosada para mejorar la falta de contorno gingival en relación a la fisura.

se planifica en base a la realización de una expansión (mínima) del maxilar para la mejora en sentido transversal de la anomalía, de acuerdo a lo conversado y coordinado con el cirujano, para posteriormente alinear y nivelar las arcadas mediante tratamiento correctivo.

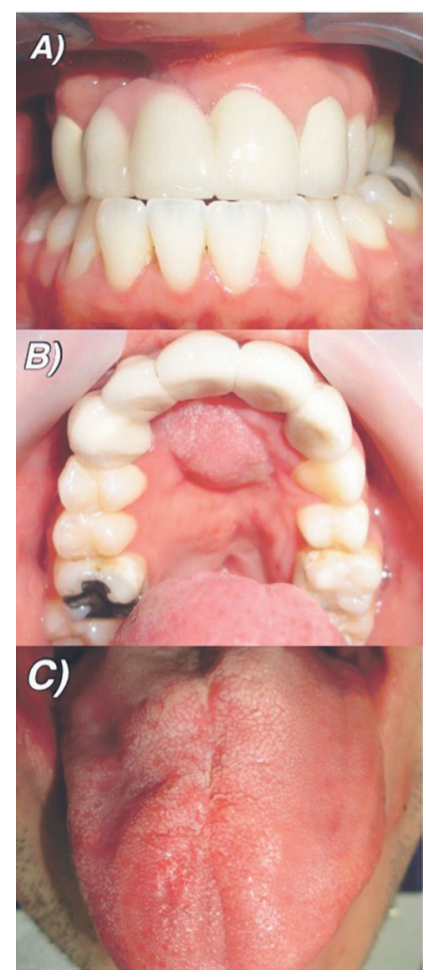

Figura 3. A. Fotografía frontal en oclusión de paciente con tratamiento finalizado. B. Injerto de mucosa lingual en zona de fístula palatina, luego de finalizado el tratamiento integral. C. Mucosa lingual posterior a cicatrización de cirugía para toma de injerto 
Se solicita evaluación en Rehabilitación Oral y Endodoncia en razón de la gran destrucción de piezas dentarias por caries. Se decide realizar endodoncia en las piezas 4.8 y 3.6, restauración de piezas 1.6, 2.6 y 2.7, más la exodoncia de la pieza 1.1 con pronóstico desfavorable por imposibilidad de participar en la rehabilitación la que, posteriormente se realizará mediante una protésica fija plural en el sector anterosuperior.

Se decide coordinar una nueva cirugía de paladar para el cierre de la fístula mediante injerto de lengua (cicatrización por $1^{\circ}$ intención) y el desligue a las 3 semanas (cicatrización por $2^{\circ}$ intención). A los 6 meses de realizada esta cirugía se decidió realizar injerto óseo alveolar derecho para lo cual se prepara ortodóncicamente la posición de dientes adyacentes.

Una vez realizado el injerto, se realiza el retiro de aparatología fija de ortodoncia y la coordinación para el inicio de la rehabilitación estética mediante prótesis fija plural, la cual utiliza como pilares las piezas 1.3, 2.1 y 2.2 para rehabilitar las piezas 1.2 y 1.1. Esto busca, además, mejorar la anatomía dentaria de los incisivos centrales superiores derecho e izquierdo y a su vez, mejorar la estética de todo el sector anterior.

Cabe mencionar que durante el período en que duró el tratamiento, fue difícil lograr una completa y correcta técnica de higiene, por lo que se sumó la aparición de nuevas caries y el posterior tratamiento de éstas.

\section{DISCUSIÓN}

Las fístulas buconasales que se generan como secuela de las cirugías de cierre de paladar en pacientes con fisura labiopalatina, suelen traer complicaciones en la fonoarticulación, en la deglución y fuertemente en el ámbito social. Los pacientes presentan dificultades durante el habla debido a la hipernasalidad de la voz y durante la ingesta de líquidos o alimentos fluidos, ya que se produce el traspaso de estos hacia la fosa nasal a través de la fístula. Por lo tanto, para aquellos pacientes que han sido sometidos a múltiples cirugías de cierre de fístula sin resultados satisfactorios, el procedimiento quirúrgico mediante un injerto de lengua se vuelve una opción factible y con buenos resultados, siempre y cuando contemos con el respaldo de un equipo multidisciplinario y con la experiencia necesaria para llevar a cabo la tarea de rehabilitar a estos pacientes.

Luego del procedimiento quirúrgico y tras realizar los controles postoperatorios, se ha observado una mantención y estabilidad del injerto en la zona de la fístula desde el momento de la cirugía a la fecha. Además, se ha producido una notable mejoría en el habla (al mejorar la IVF 9 a una IVF 2) y alimentación del paciente, influyendo positivamente en sus relaciones interpersonales y autoestima.

Entregar una solución efectiva y estable a los problemas que presentan estos pacientes producto de las fístulas buconasales debe ser nuestra prioridad al momento de la planificación del tratamiento, de tal manera de cumplir con sus expectativas y mejorar su calidad de vida.

\section{CONFLICTOS DE INTERÉS}

Los autores declaran que no existe conflicto de interés en este documento.

\section{Bibliografía}

1.Farronato G, Kairyte L, Giannini L, Galbiati G, Maspero C. How various surgical protocols of the unilateral cleft lip and palate influence the facial growth and possible orthodontic problems? Which is the best timing of the lip, palate and alveolus repair. Literature review. Stomatologija. 2014;16(2):53-60.

2. Meazzini MC, Donati V, Garattini G, Brusati R. Maxillary growth impairment in cleft lip and palate patients: a simplified aproach in the search for a cause. J Craniofac Surg. 2008;19:1302-7

3. Cohen SR, Kalinowski J, LaRossa D, Randall P. Cleft palate fistulas: A multivariate statistical analysis of prevalence, etiology, and surgical management. Plast Reconstr Surg. 1991;87:1041-7.

4. Sadhu P. Oronasal fistula in cleft palate surgery. Indian J Plast Surg 2009;42(Suppl):S123-8.

5 . Murthy J. Descriptive study of management of palatal fistula in one hundred and ninety-four cleft individuals. Indian J Plast Surg. 2011;44:41-6.

6. Chhajlani R, Ghildiyal H, Mahajan R. Role of tongue flap in palatal fistula repair: A series of 41 cases. Indian J Plast Surg. 14;47(2):210-5.
7. Posnick JC, Getz SB Jr. Surgical closure of end-stage palatal fistulas using anteriorly-based dorsal tongue flaps. J Oral Maxillofac Surg. 1987; 45: 907-912.

8. Mukherji MM Cheek flap for short palates. Cleft Palate J. 1969;6:415-20.

9. Guerrero-Santos J, Altamirano JT. The use of lingual flaps in repair of fistulas of the hard palate. Plast Reconstr Surg. 1966;38:123-8.

10. Chen HC, Ganos DL, Coessens BC, Kyutoku S, Noordhoff MS. Free forearm flap for closure of difficult oronasal fistulas in cleft palate patients. Plast Reconstr Surg. 1992;90:757-62.

11. Schwabegger AH, Hubli E, Rieger M, Gassner R, Schmidt A, Ninkovic M. Role of free-tissue transfer in the treatment of recalcitrant palatal fistulae among patients with cleft palates. Plast Reconstr Surg. 2004;113:1131-9.

12. Sendota JC, López-Noriega JC et al. Cierre de fístulas palatinas amplias mediante colgajos de lengua en pacientes con secuelas de paladar hendido. Revista Odontológica Mexicana. 2006; 10 (3): 131-137.

13. Steinhauser EW. Experience with dorsal tongue flaps for closure of defects of the hard palate. J Oral Maxillofac Surg. 1982; 40: 787-789. 